\title{
Dual ErbB1 and ErbB2 receptor tyrosine kinase inhibition exerts synergistic effect with conventional chemotherapy in pancreatic cancer
}

\author{
SMIT SINGLA $^{1}$, JAMES A. PIPPIN ${ }^{2}$ and JEFFREY A. DREBIN ${ }^{2}$ \\ ${ }^{1}$ Department of Surgery, Temple University Hospital, and ${ }^{2}$ Department of Surgery, Hospital of \\ the University of Pennsylvania, 4 Silverstein Pavilion, Philadelphia, PA 19104, USA
}

Received June 12, 2012; Accepted July 9, 2012

DOI: $10.3892 / o r .2012 .2053$

\begin{abstract}
Patient survival in pancreatic cancer remains poor with gemcitabine (GEM)-based regimens. The target specific molecular agent lapatinib, a dual ErbB1 and ErbB2 receptor tyrosine kinase inhibitor, has shown significant activity against ErbB1 and ErbB2-expressing tumors. Since pancreatic tumors frequently overexpress these proteins, we investigated its effects, both alone and in conjunction with 5-FU or GEM. The pancreatic cancer cell lines PANC-1 and AsPC were treated with varying doses of lapatinib in vitro. The effects on ErbB1/ ErbB2 protein phosphorylation and on the cell survival protein survivin were determined by western blotting. Cytotoxicity was determined by MTT assay and apoptosis was measured using the caspase-3 colorimetric assay. Similar dose-response lapatinib experiments were conducted with varying concentrations of 5-FU or GEM and isobolograms were constructed to evaluate therapeutic synergy. Lapatinib inhibited protein phosphorylation in the range of $4-16 \mu \mathrm{M}$, a clinically achievable concentration. The lapatinib-treated cells showed a dose-dependent inhibition of cell proliferation and induction of apoptosis at the same concentrations that blocked ErbB1/ ErbB2 phosphorylation. The addition of 5-FU or GEM to these cells resulted in synergistic effects. The lapatinib-treated cells also demonstrated downregulation of survivin. Simultaneous dual ErbB1 and ErbB2 receptor tyrosine kinase inhibition with lapatinib results in significant reduction of pancreatic cancer cell growth and proliferation. These effects occur at clinically achievable concentrations and are synergistic with the effects of 5-FU or GEM. These findings support the potential role of lapatinib in the treatment of pancreatic cancer.
\end{abstract}

Correspondence to: Dr Smit Singla, Department of Surgery, Temple University Hospital, 3401 N. Broad Street, 4th Floor Parkinson Pavilion, Philadelphia, PA 19140, USA

E-mail: smit.singla@tuhs.temple.edu

Key words: ErbB1, ErbB2, pancreas cancer, lapatinib

\section{Introduction}

Pancreatic cancer is the fourth leading cause of cancer death in both men and women in the United States. It has amongst the worst prognoses of all human malignancies. A majority of patients are symptomatic at the time of diagnosis and have developed advanced metastatic disease (1). Most patients die within a year of diagnosis and the 5-year survival rate is $<5 \%$ (2). Despite many multi-modal treatment strategies, the survival rates have not improved significantly and mortality remains high (3).

Gemcitabine (GEM) has remained the mainstay primary chemotherapeutic agent for systemic treatment in advanced pancreatic adenocarcinoma. Multiple studies have shown survival benefits of GEM chemotherapy, both alone as well as in combination with other drugs (4-6). However, despite significant advances and improvement in therapy, the 5-year survival remains quite low. As a result, there is now a shift towards target specific agents, in addition to conventional cytotoxic drugs, with an aim towards increasing overall survival (7-11).

ErbB1 and ErbB2 are members of the epidermal growth factor (EGF) receptor family and are known to play an essential role in regulation of cell proliferation and differentiation. The formation of homodimers and heterodimers between different EGF receptors is believed to regulate complex signal transduction pathways via activation of intrinsic protein tyrosine kinase activity. This leads to recruitment and phosphorylation of several intracellular substrates leading to various cellular activities including mitogenic signaling and cell growth $(12,13)$.

Some studies have also suggested that overexpression of ErbB1 and ErbB2 in certain cancers is associated with increased tumor aggression and poor prognosis $(12,14,15)$. Targeted downregulation of these receptors has been shown to cause apoptosis and cell death in cancers that overexpress these receptors (12). Since a significant percentage of pancreatic tumors overexpress both ErbB1 and ErbB2 (14-16), targeting these receptors may be a viable strategy in patients diagnosed with such tumors.

Many studies have looked at the response of pancreatic neoplasms to ErbB1 or ErbB2 targeted therapy. Transtuzumab 


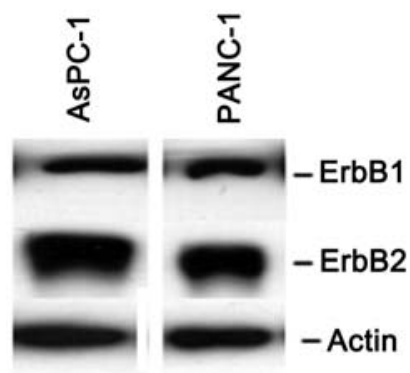

Figure 1. Immunoblotting assay, ErbB1 and ErbB2 protein expression in AsPC and PANC-1 cell lines.

(ErbB2) (11), erlotinib (ErbB1) (10), cetuximab (ErbB1) (8) and gefitinib (ErbB1) (9) have been studied in the last few years; however, none of these drugs has shown any significant improvement in mortality when compared to treatment with conventional cytotoxic drugs. It was observed that most patients eventually developed drug resistance. This negated any benefits that these agents potentially offered. Since, all these studies targeted either ErbB1 or ErbB2 separately, we hypothesized that simultaneous inhibition of both ErbB1 and ErbB2 receptors might suppress tumor growth better than targeting them individually, and may help overcome the development of drug resistance. We selected lapatinib (Tykerb), a dual ErbB1 and ErbB2 tyrosine kinase enzyme inhibitor, for our study since lapatinib has been shown in multiple studies to benefit patients who have developed drug resistance to prior targeted therapies (17-19).

\section{Materials and methods}

Pancreatic cell lines and culture. Human pancreatic cell lines AsPC-1 and PANC-1 were selected for our experiments due to their expression of ErbB1 and ErbB2 receptors (Fig. 1). All cell lines were purchased from ATCC (Manassas, VA) and maintained in recommended media supplemented with $10 \%$ fetal bovine serum (Mediatech, Manassas, VA). All cells were cultured at $37^{\circ} \mathrm{C}$ in $5 \% \mathrm{CO}_{2}$ with $100 \%$ humidity.

Chemicals. Lapatinib (Tykerb, Genentech Inc, South San Francisco, CA) was kindly provided by Genentech Inc. (South San Francisco, CA) and gemcitabine and 5-fluorouracil (5-FU) were obtained from Sigma/Aldrich, St. Louis, MO.

In vitro proliferation assay (MTT assay). Cell growth was assessed using a 3-(4,5-dimethylthiazol-2-yl)-2,5-diphenyltetrazolium bromide assay (MTT assay). Briefly, 5,000 viable cells were seeded into flat-bottomed 96 -well plates in triplicate and allowed to adhere overnight. Cells were treated with the addition of the intended doses of lapatinib $(0-16 \mu \mathrm{M})$, gemcitabine (0-400 $\mathrm{nM})$ and/or 5-FU (0-16 $\mu \mathrm{M})$. After 3-4 days of incubation, 3-(4,5-dimethylthiazol-2-yl)-2,5-diphenyltetrazolium bromide was added to a final concentration of $0.5 \mathrm{mg} / \mathrm{ml}$ and cells were incubated an additional $4 \mathrm{~h}$. Cells were lysed with the addition of isopropanol. Absorbance was measured at 595 and $655 \mathrm{~nm}$ in a 96-well plate reader (BioRad Model 680, Bio-Rad Laboratories, Hercules, CA) and growth curves calculated.
In vitro apoptosis assay. Apoptosis activity was assessed using the ApoAlert Caspase-3 Colorimetric Assay kit (Clontech, Mountain View, CA). Briefly, $10^{6}$ viable cells were seeded in $100-\mathrm{mm}$ tissue culture plates and allowed to adhere overnight. Cells were treated with the addition of the intended doses of lapatinib $(0-16 \mu \mathrm{M})$, gemcitabine $(0-400 \mathrm{nM})$ and/or 5-FU $(0-16 \mu \mathrm{M})$. Cells were harvested from plates using cell scrapers $48 \mathrm{~h}$ after the addition of drugs and lysed for analysis of caspase-3 activity according to the manufacturer's instructions. Readings were taken at $405 \mathrm{nM}$ in disposable cuvettes in a spectrophotometer (Thermogenesis 6, Hopkinton, MA) and caspase-3 activity was calculated.

In vitro immunoblot analyses (western blot analysis). Cells were plated and treated in the same manner as described for the apoptosis assay. After incubating for $48 \mathrm{~h}$, cells were harvested using cell scrapers and washed with DPBS. Cells were lysed on ice in HEPES lysis buffer containing $50 \mathrm{mM}$ HEPES, $100 \mathrm{mM} \mathrm{NaCl}, 1 \%$ Triton X-100, 10\% Glycerol, $1 \mathrm{mM}$ $\mathrm{Na}_{3} \mathrm{VO}_{4}, 5 \mathrm{mM} \mathrm{NaFl}, 1: 50$ dilution of Complete Protease Inhibitor Cocktail tablets (Roche, Indianapolis, IN). Protein concentrations were determined via Coomassie Protein Assay Reagent (Thermo Scientific, Rockford, IL) and samples were standardized to $10 \mu \mathrm{g}$ in SDS-PAGE loading buffer. Samples were loaded on a $7.5 \%$ SDS-PAGE acrylamide gel and run under reducing conditions for $2 \mathrm{~h}$ at $100 \mathrm{~V}$. The proteins were transferred to Immobilon-P membranes (Millipore Co., Billerica, MA) using Towbins transfer buffer ( $25 \mathrm{mM}$ Tris, $192 \mathrm{mM}$ glycine, $10 \%$ methanol). Membranes were blocked in TBS (20 mM Tris, $150 \mathrm{mM} \mathrm{NaCl}$ ) containing $0.1 \%$ Tween-20 and 5\% non-fat dry milk or BSA and incubated with antibodies against target proteins. Antibodies include ErbB1 (Chemicon, Temecula, CA), ErbB2 (AbCam, Cambridge, MA), Phos-ERBB1 (AbCam), Phos-ERBB2 (Cell Signaling, Beverly, MA), $\alpha$-tubulin (Santa Cruz Biotechnology, Santa Cruz, CA).

\section{Results}

The effects of lapatinib on cell proliferation. Initial experiments studied the effects of lapatinib, both alone and in combination with 5-FU or gemcitabine, on proliferation of AsPC and PANC-1 cells in vitro. Lapatinib inhibited cell proliferation in a dose-dependent manner. At lower doses (0.25-1 $\mu \mathrm{M})$ lapatinib had no effect, but at higher doses (4-16 $\mu \mathrm{M})$, inhibition was significant. There was a 50\% inhibition in cell proliferation at a dose of $12 \mu \mathrm{M}$, but the effects plateaued at higher doses (Fig. 2).

The inhibitory effects of lapatinib were potentiated in the presence of 5-FU or GEM, with a dose-dependent shift in the proliferation curve (Figs. 3 and 4). This suggests that lapatinib may work synergistically with these other drugs, increasing their effect in vitro. The combination of drugs allowed similar inhibition at much lower doses. This may allow for reducing the dosage of each drug in vivo and thus the incidence of adverse side effects often noticed in current recommended dosages $(20,21)$.

The effect of lapatinib on ErbB1 and ErbB2 protein levels. AsPC and PANC-1 cells were treated with lapatinib at dosages 


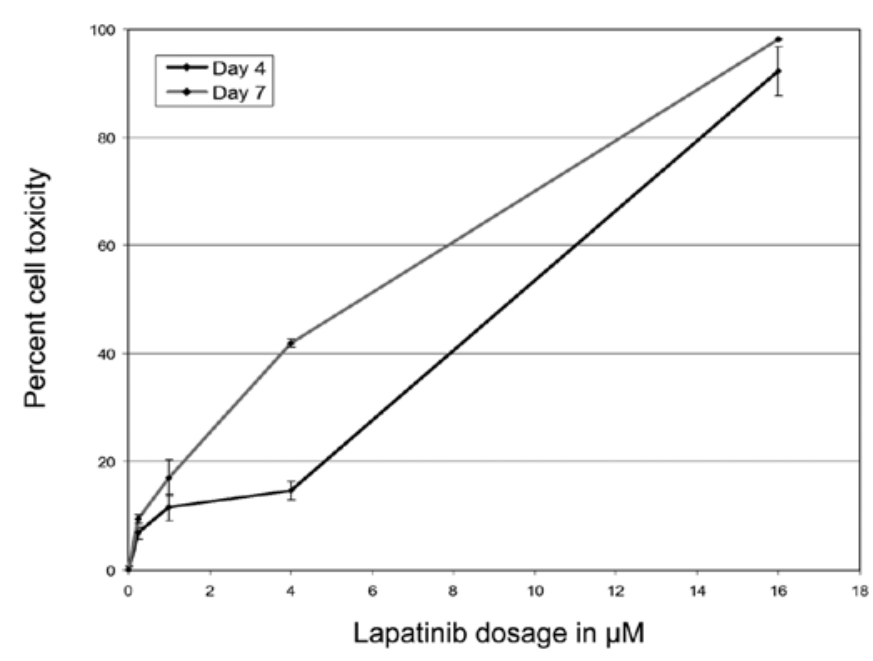

Figure 2. MTT assay. Effect of lapatinib on cytotoxicity. Treatment of cells with lapatinib in vitro caused a dose-dependent increase in cellular toxicity.

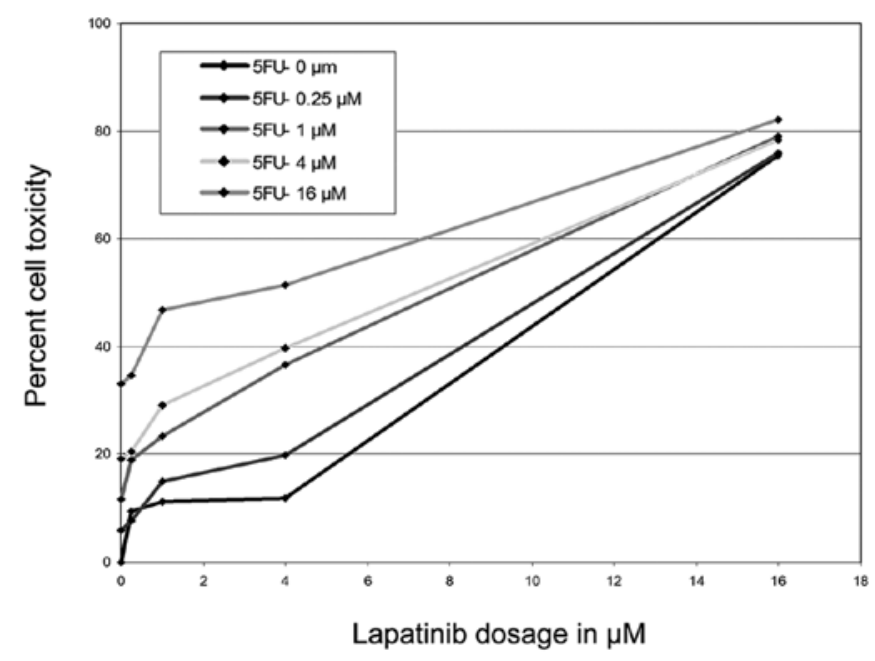

Figure 3. MTT assay. Effect of combining lapatinib with 5-FU on cytotoxicity.

similar to those used in the cell proliferation assays. Following treatment with lapatinib, samples were analyzed for expression of ErbB1, ErbB2 and their respective phosphorylated proteins using immunoblotting.

While the levels of total ErbB1 and ErbB2 proteins remained unchanged, both phosphorylated ErbB1 and ErbB2 (pErbB1-Tyr1173 and ErbB2-Tyr1248) protein levels decreased with lapatinib treatment. Again, lower doses showed no effects; however, at higher doses $(12-16 \mu \mathrm{M})$ decrease in phosphorylated protein was detectable. There was an estimated $50 \%$ reduction in phosphorylated protein at $12 \mu \mathrm{M}$, which corresponds to the dose needed to inhibit proliferation by the same amount (Fig. 5). These sites (Tyr1173 and Tyr1248) have been found to be involved in regulation of tyrosine kinase activity and phosphorylation of these sites couples ErbB1 and ErbB2 to downstream kinase signal transduction pathways (22-24). Therefore, lapatinib downregulation of phosphorylated ErbB1 and ErbB2 may decrease tyrosine kinase activity and subsequently decrease proliferation through these downstream pathways.

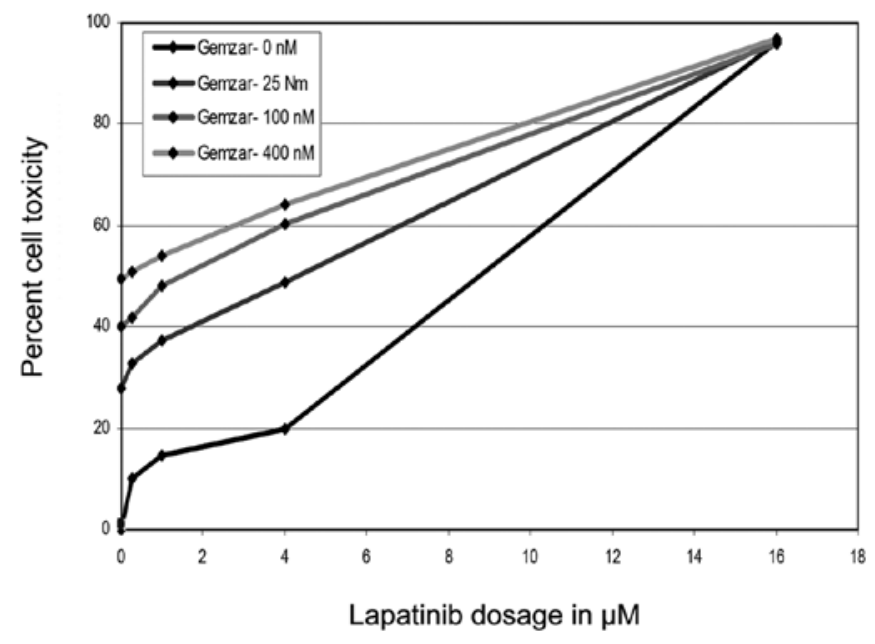

Figure 4. MTT assay. Effect of combining lapatinib with gemcitabine on cytotoxicity.

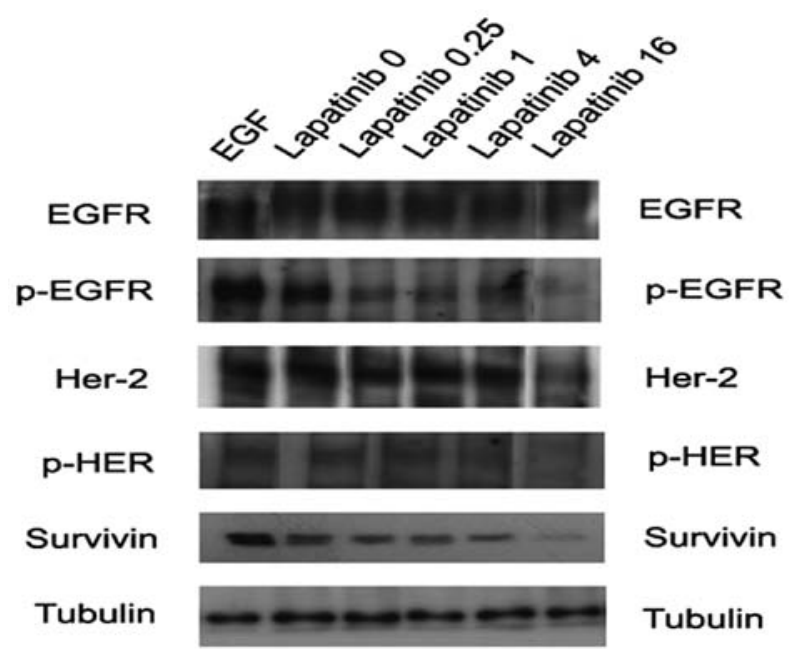

Figure 5. Immunoblotting assay. Effects of lapatinib on ErbB protein phosphorylation and survivin expression. Treatment of cells with lapatinib in vitro caused a dose-dependent downregulation of phospho-proteins and survivin.

The effect of lapatinib on cell apoptosis. AsPC and PANC-1 cells were treated in the same manner as the proliferation assays. Forty-eight hours after treatment, apoptosis activity was evaluated using a caspase -3 and -8 colorimetric assay kit. Both cell lines exhibited an increase in cleaved caspase-3 activity indicating apoptosis. This activity corresponded with doses showing decreased proliferation with the maximal activity in the range of 4-16 $\mu \mathrm{M}$ (Fig. 6). This would suggest that lapatinib is causing apoptosis in addition to decreasing proliferation.

The effect of lapatinib on survivin protein level. Based on the apoptosis results, we decided to test survivin levels under our treatment conditions. Survivin has been shown to inhibit apoptosis by binding to caspase-3 (25) and has also been shown to play a role in pancreatic cancer (26-28). In order to determine if lapatinib affects the survivin pathway, the expression of survivin protein was assessed in both AsPC and PANC-1 cells 


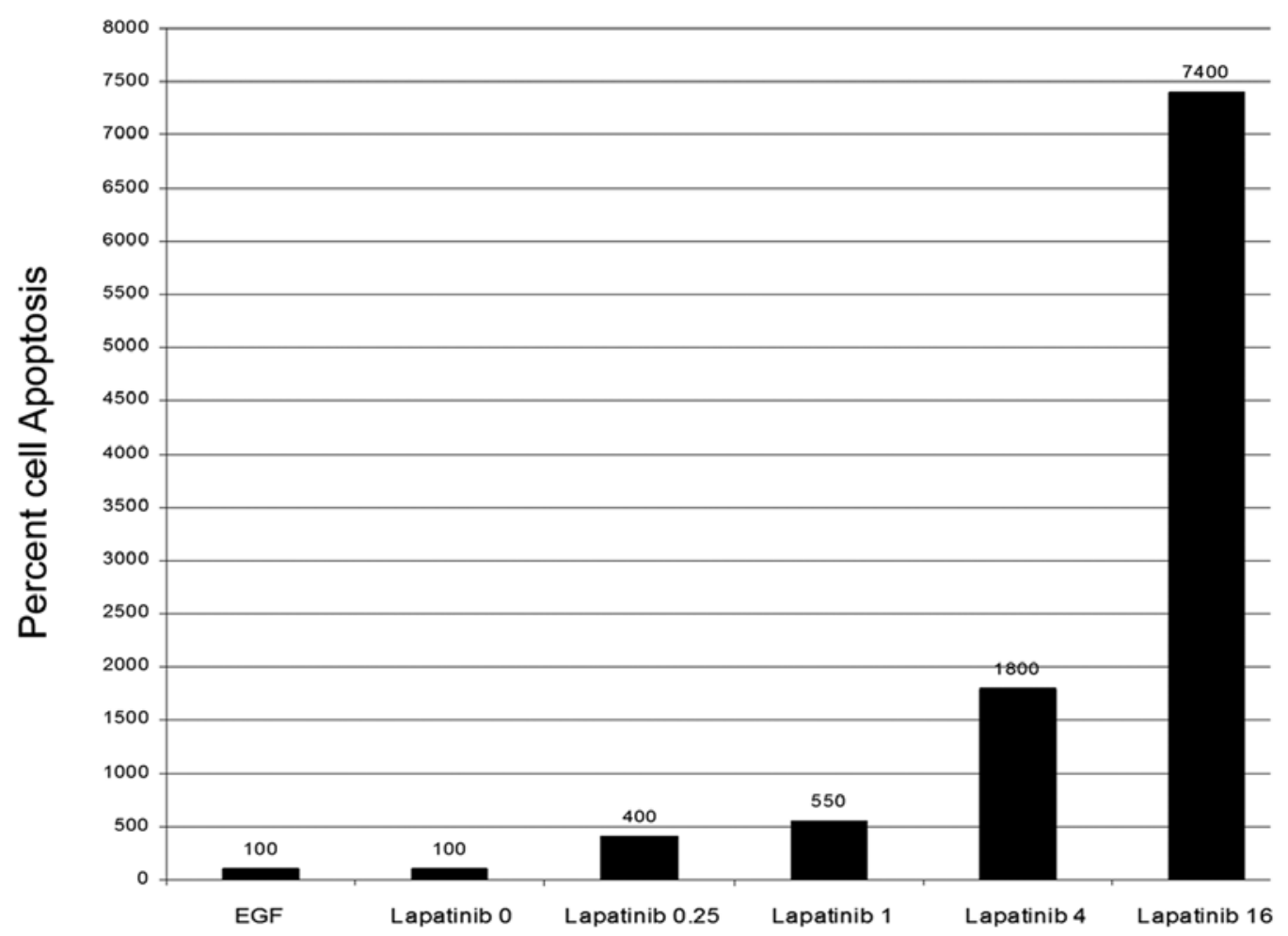

Figure 6. Caspase-3 colorimetric assay. Treatment of AsPC cells with lapatinib (dose in $\mu \mathrm{M}$ ) in vitro caused a dose-dependent increase in cell apoptosis.

using immunoblotting. Again, there was a dose-dependent decrease in expression of survivin protein levels with lapatinib treatment in both cell lines. AsPC cells showed a complete reduction of expression at $12 \mu \mathrm{M}$, while PANC-1 cells showed only a 50\% reduction at this same dose (Fig. 5). Assuming survivin is inhibiting apoptosis in these cells; lapatinib could play a key role restoring apoptosis in pancreatic cancer cells.

\section{Discussion}

Pancreatic tumors have so far shown substantial resistance to multiple chemotherapeutic drugs and regimens. It has become a major challenge for clinicians to offer substantive treatment options to patients with advanced pancreatic carcinomas. However, with recent advancements in treatment strategies directed against molecular targets, there has been a renewed interest. It has been proposed that novel molecular agents targeting ErbB1 and ErbB2 in pancreatic tumors will help overcome substantial tumor resistance against conventional cytotoxic drugs and also offer patient specific therapy depending upon the receptor and molecular subtype.

As discussed earlier, some pancreatic tumors have high levels of ErbB1 and ErbB2 cell surface receptors (14-16). These tumors, therefore, are ideal for targeted therapeutic strategy since both ErbB1 and ErbB2 receptors along with their downstream proteins have been shown to promote cell growth and survival, and mediate resistance to chemotherapy. Many studies have been published describing the effects of newer molecular agents directed against ErbB1 and ErbB2 receptors in pancreatic neoplasms (8-11), however, despite theoretical advantages; these agents have failed to yield significantly beneficial results, either alone or in combination with conventional cytotoxic drugs. Although, the exact mechanisms responsible for failure to respond are a matter of intense scrutiny, it seems that a significant number of patients develop drug resistance. Numerous studies have been published describing the potential mechanisms of resistance against these agents. Engelman et al (29) showed that amplification of MET caused gefitinib resistance by driving HER3-dependent activation of PI3K while Scaltriti et al (17) found that expression of truncated HER2 altered the ErbB2 receptor such that it impaired the access of trastuzumab to the attachment site. Furthermore, Nagy et al (30) described the development of resistance in some patients due to overexpression of MUC4.

While it remains to be seen what exactly causes development of resistance against these agents, there is ample evidence to suggest that ErbB subunits work in tandem as a unit and therefore, it may be possible that broad inhibition of these subunits may be necessary to overcome the development of resistance. Working on this hypothesis, we selected lapatinib, based on its mechanism of dual inhibition of ErbB1 and ErbB2 tyrosine kinase and its favorable response in patients who have developed resistance against single-receptor based molecular agents (17,31).

We found that lapatinib markedly reduced tyrosine phosphorylation of ErbB1 and ErbB2, which inhibits activation of Erk1/2 and AKT, the downstream effectors of cell proliferation and survival, respectively (32). We also found that lapatinib caused a decrease in caspase- 3 activity and the expression of survivin, a protein inhibitor of the apoptosis. This is significant because the exact mechanism by which lapatinib exerts its apoptotic effect is unknown and therefore, it may be possible that the inhibition of survivin could be one of mechanisms by which lapatnib exerts its effects. Many studies have shown that 
survivin protects tumors from programmed cell death (33-35), however, none of the prior studies with either ErbB1 or ErbB2 inhibitors could modulate survivin.

Investigators have added lapatinib to chemotherapeutic agents such as paclitaxel (36), docetaxel (37), capecitabine (38), letrozole (39), and to combination regimens such as FOLFOX (40) and FOLFOX (41), with promising results. Although clinical trials in different tumor types are ongoing, the most mature data thus far have been in the treatment of breast cancer, particularly in trastuzumab-resistant patients (37). We also studied the effects of lapatinib in combination with 5-FU and gemcitabine. In combination, lapatinib revealed enhanced toxicity with pronounced cell death and decreased cell survival. These effects were consistent in both cell lines suggesting that its effects may be broad based in cells expressing these target proteins.

In conclusion, simultaneous dual ErbB1 and ErbB2 receptor tyrosine kinase inhibition with lapatinib inhibits pancreatic cancer cell growth and proliferation and induces apoptotic cell death. These effects occur at clinically achievable concentrations and are synergistic with the effects of conventional chemotherapy agents including 5-FU or gemcitabine. These findings support the potential role of lapatinib in the treatment of pancreatic cancer either alone or as an adjunct to conventional chemotherapy agents.

\section{References}

1. Nelson NJ: Pancreatic cancer research matures. J Natl Cancer Inst 99: 1432-1434, 2007.

2. Hidalgo M: Pancreatic cancer. N Engl J Med 362: 1605-1617, 2010.

3. Li J and Saif MW: Any progress in the management of advanced pancreatic cancer? JOP 10: 361-365, 2009.

4. Zhu CP, Shi J, Chen YX, Xie WF and Lin Y: Gemcitabine in the chemoradiotherapy for locally advanced pancreatic cancer: a meta-analysis. Radiother Oncol 99: 108-113, 2011.

5. Heinemann V, Boeck S, Hinke A, Labianca R and Louvet C: Meta-analysis of randomized trials: evaluation of benefit from gemcitabine-based combination chemotherapy applied in advanced pancreatic cancer. BMC Cancer 8: 82, 2008.

6. Yip D, Karapetis C, Strickland A, Steer CB and Goldstein D: Chemotherapy and radiotherapy for inoperable advanced pancreatic cancer. Cochrane Database Syst Rev 3: CD002093, 2006.

7. Rivera F, Lopez-Tarruella S, Vega-Villegas ME and Salcedo M: Treatment of advanced pancreatic cancer: from gemcitabine single agent to combinations and targeted therapy. Cancer Treat Rev 35: 335-339, 2009.

8. Philip PA, Benedetti J, Corless CL, et al: Phase III study comparing gemcitabine plus cetuximab versus gemcitabine in patients with advanced pancreatic adenocarcinoma: Southwest Oncology Group-directed intergroup trial S0205. J Clin Oncol 28: 3605-3610, 2010.

9. Fountzilas G, Bobos M, Kalogera-Fountzila A, et al: Gemcitabine combined with gefitinib in patients with inoperable or metastatic pancreatic cancer: a phase II Study of the Hellenic Cooperative Oncology Group with biomarker evaluation. Cancer Invest 26: 784-793, 2008

10. Moore MJ, Goldstein D, Hamm J, et al: National Cancer Institute of Canada Clinical Trials Group. Erlotinib plus gemcitabine compared with gemcitabine alone in patients with advanced pancreatic cancer: a phase III trial of the National Cancer Institute of Canada Clinical Trials Group. J Clin Oncol 25: 1960-1966, 2007.

11. Safran H, Iannitti D, Ramanathan R, et al: Herceptin and gemcitabine for metastatic pancreatic cancers that overexpress ERBB2/neu. Cancer Invest 22: 706-712, 2004.

12. Mendelsohn J and Baselga J: The EGF receptor family as targets for cancer therapy. Oncogene 19: 6550-6565, 2000.
13. Riese DJ II and Stern DF: Specificity within the EGF family/ ErbB receptor family signaling network. Bioessays 20: 41-48, 1998.

14. Ueda S, Ogata S, Tsuda H, et al: The correlation between cytoplasmic overexpression of epidermal growth factor receptor and tumor aggressiveness: poor prognosis in patients with pancreatic ductal adenocarcinoma. Pancreas 29: e1-e8, 2004.

15. Dancer J, Takei H, Ro JY and Lowery-Nordberg M: Coexpression of ERBB1 and ERBB2 in pancreatic ductal adenocarcinoma: a comparative study using immunohistochemistry correlated with gene amplification by fluorescencent in situ hybridization. Oncol Rep 18: 151-155, 2007.

16. Tobita K, Kijima H, Dowaki S, et al: Epidermal growth factor receptor expression in human pancreatic cancer: significance for liver metastasis. Int J Mol Med 11: 305-309, 2003.

17. Scaltriti M, Rojo F, Ocana A, et al: Expression of p95HER2, a truncated form of the HER2 receptor, and response to anti-HER2 therapies in breast cancer. J Natl Cancer Inst 99: 628-638, 2007

18. Konecny GE, Pegram MD, Venkatesan N, et al: Activity of the dual kinase inhibitor lapatinib (GW572016) against HER-2overexpressing and trastuzumab-treated breast cancer cells. Cancer Res 66: 1630-1639, 2006.

19. O'Brien NA, Browne BC, Chow L, et al: Activated phosphoinositide 3-kinase/AKT signaling confers resistance to trastuzumab but not lapatinib. Mol Cancer Ther 9: 1489-1502, 2010.

20. Conroy T, Desseigne F, Ychou M, et al: FOLFIRINOX versus gemcitabine for metastatic pancreatic cancer. N Engl J Med 364: 1817-1825, 2011.

21. Mehta SP: Palliative chemotherapy for pancreatic malignancies. Surg Clin North Am 90: 365-375, 2010.

22. Muthuswamy SK, Gilman M and Brugge JS: Controlled dimerization of ErbB receptors provides evidence for differential signaling by homo- and heterodimers. Mol Cell Biol 19: 6845-6857, 1999

23. Zwick E, Hackel PO, Prenzel N and Ullrich A: The EGF receptor as central transducer of heterologous signalling systems. Trends Pharmacol Sci 20: 408-412, 1999.

24. Sorkin A, Helin K, Waters CM, Carpenter G and Beguinot L: Multiple autophosphorylation sites of the epidermal growth factor receptor are essential for receptor kinase activity and internalization. Contrasting significance of tyrosine 992 in the native and truncated receptors. J Biol Chem 267: 8672-8678, 1992.

25. Li F, Ambrosini G, Chu EY, Plescia J, Tognin S, Marchisio PC and Altieri DC: Control of apoptosis and mitotic spindle checkpoint by survivin. Nature 396: 580-584, 1998 .

26. Liu BB and Wang WH: Survivin and pancreatic cancer. World J Clin Oncol 2: 164-168, 2011.

27. Theodoropoulos GE, Michalopoulos NV, Panoussopoulos SG, Taka S and Gazouli M: Effects of caspase-9 and survivin gene polymorphisms in pancreatic cancer risk and tumor characteristics. Pancreas 39: 976-980, 2010.

28. Shen YM, Yang XC, Song ML, Qin CH, Yang C and Sun YH: Growth inhibition induced by short hairpin RNA to silence survivin gene in human pancreatic cancer cells. Hepatobiliary Pancreat Dis Int 9: 69-77, 2010.

29. Engelman JA, Zejnullahu K, Mitsudomi T, et al: MET amplification leads to gefitinib resistance in lung cancer by activating ERBB3 signaling. Science 316: 1039-1043, 2007.

30. Nagy P, Friedlander E, Tanner M, Kapanen AI, Carraway KL, Isola J and Jovin TM: Decreased accessibility and lack of activation of ErbB2 in JIMT-1, a herceptin-resistant, MUC4-expressing breast cancer cell line. Cancer Res 65: 473-482, 2005.

31. Xia W, Liu LH, Ho P and Spector NL: Truncated ErbB2 receptor (p95ErbB2) is regulated by heregulin through heterodimer formation with ErbB3 yet remains sensitive to the dual EGFR/ ErbB2 kinase inhibitor GW572016. Oncogene 23: 646-653, 2004.

32. Xia W, Mullin RJ, Keith BR, et al: Anti-tumor activity of GW572016: a dual tyrosine kinase inhibitor blocks EGF activation of EGFR/erbB2 and downstream Erk1/2 and AKT pathways. Oncogene 21: 6255-6263, 2002.

33. Song Z, Yao X and Wu M: Direct interaction between survivin and Smac/DIABLO is essential for the anti-apoptotic activity of survivin during taxol-induced apoptosis. J Biol Chem 278: 23130-23140, 2003.

34. Wang Z, Fukuda S and Pelus LM: Survivin regulates the p53 tumor suppressor gene family. Oncogene 23: 8146-8153, 2004. 
35. Desplanques G, Hulin P, Juin P and Barille-Nion S: Survivin Protects Breast Cancer Cells from DNA Damage during Mitosis (abstract). In: Thirty-Second Annual CTRC-AACR San Antonio Breast Cancer Symposium, Dec 10-13; San Antonio, TX, 2009. Cancer Res 69 (Suppl 3), 2009.

36. Boussen H, Cristofanilli M, Zaks T, DeSilvio M, Salazar V and Spector N: Phase II study to evaluate the efficacy and safety of neoadjuvant lapatinib plus paclitaxel in patients with inflammatory breast cancer. J Clin Oncol 28: 3248-3255, 2010.

37. LoRusso PM, Jones SF, Koch KM, et al: Phase I and pharmacokinetic study of lapatinib and docetaxel in patients with advanced cancer. J Clin Oncol 26: 3051-3056, 2008.

38. Cameron D, Casey M, Press M, et al: A phase III randomized comparison of lapatinib plus capecitabine versus capecitabine alone in women with advanced breast cancer that has progressed on trastuzumab: updated efficacy and biomarker analyses. Breast Cancer Res Treat 112: 533-543, 2008.
39. Johnston S, Pippen J Jr, Pivot X, et al: Lapatinib combined with letrozole versus letrozole and placebo as first-line therapy for postmenopausal hormone receptor-positive metastatic breast cancer. J Clin Oncol 27: 5538-5546, 2009.

40. Siegel-Lakhai WS, Beijnen JH, Vervenne WL, et al: Phase I pharmacokinetic study of the safety and tolerability of lapatinib (GW572016) in combination with oxaliplatin/fluorouracil/ leucovorin (FOLFOX4) in patients with solid tumors. Clin Cancer Res 13: 4495-4502, 2007.

41. Midgley RS, Kerr DJ, Flaherty KT, et al: A phase I and pharmacokinetic study of lapatinib in combination with infusional 5-fluorouracil, leucovorin and irinotecan. Ann Oncol 18: 2025-2029, 2007. 